\title{
Special Issue on "Advances in Condition Monitoring, Optimization and Control for Complex Industrial Processes"
}

\author{
Zhiwei Gao $^{1, *(\mathbb{D})}$, Michael Z. Q. Chen ${ }^{2} \mathbb{D}$ and Dapeng Zhang ${ }^{3}$ \\ 1 Faculty of Engineering and Environment, University of Northumbria at Newcastle, \\ Newcastle upon Tyne NE1 8ST, UK \\ 2 School of Automation, Nanjing University of Science and Technology, Nanjing 210094, China; \\ mzqchen@njust.edu.cn \\ 3 School of Electrical Engineering and Automation, Tianjin University, Tianjin 300072, China; zdp@tju.edu.cn \\ * Correspondence: zhiwei.gao@northumbria.ac.uk
}

check for

updates

Citation: Gao, Z.; Chen, M.Z.Q.; Zhang, D. Special Issue on "Advances in Condition Monitoring, Optimization and Control for Complex Industrial Processes". Processes 2021, 9, 664. https:// doi.org/10.3390/pr9040664

Received: 8 April 2021

Accepted: 8 April 2021

Published: 9 April 2021

Publisher's Note: MDPI stays neutral with regard to jurisdictional claims in published maps and institutional affiliations.

Copyright: (c) 2021 by the authors. Licensee MDPI, Basel, Switzerland. This article is an open access article distributed under the terms and conditions of the Creative Commons Attribution (CC BY) license (https:// creativecommons.org/licenses/by/ $4.0 /)$.
Complex industrial automation systems and processes, such as chemical processes, manufacturing systems, wireless network systems, power and energy systems, smart grids and so forth, have greatly contributed to our daily life. Complex engineering systems are rather expensive, with a high requirement for system reliability and control and production performance [1,2]. As a result, there has been increasing demand in the complex industries to develop reliable condition-monitoring techniques to monitor real-time system status and promote advanced optimization algorithms and resilient control methods to ensure the desired control and operation performance. Recently, artificial intelligence, data-driven techniques, cyber-physical systems, and cloud and cognitive computation have further stimulated research and applications of monitoring, optimization, and control techniques.

This Special Issue aims to provide a platform for researchers and engineers to report their recent results, exchange research ideas, and have an overview of emerging research and application directions in condition monitoring, optimization, and advanced control for complex industrial processes. There are 25 papers included in this Special Issue after a rigorous review process, which are presented in Table 1 according to their categories.

Table 1. Categories of the papers included in the Special Issue.

\begin{tabular}{cccc}
\hline Categories & $\begin{array}{c}\text { Condition } \\
\text { Monitoring \& } \\
\text { Resilient Strategies }\end{array}$ & $\begin{array}{c}\text { Control } \\
\text { Applications }\end{array}$ & Optimization \\
\hline Papers & {$[3-11]$} & {$[12-20]$} & {$[21-27]$} \\
\hline
\end{tabular}

\section{Condition Monitoring and Resilient Strategies for Complex Industrial Processes}

Condition monitoring is the process of monitoring system parameters in an industrial process to identify a significant change that is indicative of a developing fault. Condition monitoring can be regarded as fault detection, which can tell whether the system is healthy and when a fault occurs. Along with condition monitoring and fault detection, fault isolation is used to find out which component is faulty, and fault identification aims to determine the size and type of the faults, which is important evaluation on the severity degree of a fault. Prognosis aims to predict the remaining useful life of a system or component. Condition monitoring, fault diagnosis and prognosis play a key role for predictive maintenance. Resilient methods are used to accommodate the faults so that the system can operate with a tolerant performance degradation. As a result, condition monitoring and resilience strategies are paramount to improve the reliability, safety, availability, and productiveness of an industrial automation process. Gao and Liu in [3] provided a comprehensive overview on condition monitoring, fault diagnosis, and prognosis and resilient design on wind turbine energy systems. Condition monitoring and fault diagnosis 
approaches were reviewed following the categories of model-based approaches, signalbased methods, knowledge-based techniques, and the hybrid of the approaches above. Prognosis methods were surveyed following the classes of model-based and data-based methods, and their combination. Resilience strategies were overviewed based on the sets of passive and active techniques and their combinations. The comparisons on different techniques were discussed, and their advantages and disadvantages were commented on. Both gear-box coupled generator-based (i.e., doubly fed induction generators) wind turbine systems and direct-drive generator-based (e.g., permanent-magnet synchronous generators) wind turbine systems were reviewed in an unified framework. An overview of further research directions in this field was also provided. The comprehensive survey paper with 106 references will much benefit the researchers and engineers so that they can get insight into this field conveniently.

Fu et al., in [4] integrated fast Fourier transform (FFT) and uncorrelated multilinear principal component analysis (UMPCA) techniques for fault detection and classification of a 4.8 MW wind turbine benchmark system under five faulty scenarios. By using the detailed comparison studies, the effectiveness of the proposed algorithm was illustrated and demonstrated. It concluded that, among all the used algorithms in this paper, the FFT showed a significantly positive impact on the improvement of the performance of the fault diagnosis and classification. Moreover, it showed that the proposed FFT plus UMPCA algorithm can also recognize the differences between the data within the same class.

A control chart plays an important role in production processes for monitoring the quality of the products and preventing defects. Aslam et al., in [5] addressed a controlchart algorithm to monitor the mean time between two events using a belief estimator under the neutron-sophic gamma distribution. The proposed control chart approach was demonstrated to be more effective to detect the causes of the variations in the process than the conventional chart using classical statistics under uncertain environments.

In [6], Xie et al., determined the key parameters of the Holmquist-Johnson-Cook (HJC) constitutive model for coal by using a series of experimental tests, which were important to understand the occurrence mechanism and predict coal-rock dynamic disasters. By implementing split Hopkinson pressure bar (SHPB) experiments and simulation studies on the impact damage of the coal using ANSYS/LS-DYNA software, the reliability of the HJC constitutive model parameters for briquette were analyzed and validated. The HJC constitutive model parameters were used to analyze impact damage of the tunnel face in simulations; it was shown that the failure process of the coal seam in the roadway was visually present. The results of this paper would benefit understanding of the mechanism of coal-rock dynamic disasters better.

Operation conditions of the flotation process can be reflected by the features of the froth image in a zinc flotation process, where the bubble size is the most evident feature. In the paper authored by Tang et al. [7], the bubble size cumulative distribution function was adopted as a new feature of the froth image, and the estimation approach for the cumulative distribution function of the bubble sizes was addressed. The froth image features would change continuously with time, caused by the change of the reagent dosage. The relationship between the time series of the bubble size cumulative distribution function and the reagent dosage was analyzed, and a nonlinear relationship model between the dynamic feature vectors and the dosage of reagent in the flotation process was established. The industrial experiment results demonstrated the effectiveness of the proposed approach for operation condition monitoring.

It is important but challenging to find out the load state of a wet ball mill during the grinding process. In the work by Cai et al. [8], a novel approach for mill load identification was proposed by synthesizing empirical wavelet transform, multiscale fuzzy entropy, and adaptive evolution particle swarm optimization probabilistic neural network classification. The feasibility of the presented method was verified using grinding experiments, which showed that overall recognition rate was as high as $97.3 \%$, outperforming the existing 
methods in the literature. It concluded that the addressed method can accurately identify and monitor different load states of a ball mill.

Taking the Xiashanmao coal mine as an engineering object, the stress distribution coal seam mining process was investigated by Sun et al. [9]. Using the new technology of gob-side entry retaining by roof cutting without pillar, the mechanical model of the roof structure was established. Implementing numerical simulation, the distributions of strike and lateral abutment pressure of the working faces were obtained. Mine pressure monitoring data were used to verify the proposed methods, showing the consistency between the simulated results and monitoring results.

Modern power systems are usually complex and high-nonlinear, which can be modelled as directed graphs. The graphs can be divided into communities, and it is thus of interest to find out an optimal community to alleviate cascading failure propagation. In [10], $\mathrm{Hu}$ and Lee proposed three low-degree-node-based link-addition strategies to optimize the original topology, by considering islanding characteristics and node vulnerability. An evaluation index was addressed to measure impacts from sequential attacks on the network. The results of this paper would benefit design of an optimal power network to mitigate power system cascading failures.

Modern industrial systems are prone to cyber-attacks due to potential vulnerabilities of the underlying entities in systems. It is important for a system to have resilience so that it can recover to a normal state under attacks. In the paper authored by Ibrahim and Alsheikh [11], a hybrid attack graph was introduced to deal with system resilience under various attacks. By using the hybrid attack graph, the evolution of both logical and real values of system parameters can be captured under attack and recovery actions, where the hybrid attack graph was generated automatically and visualized using Java-based tools. The effectiveness of the results was illustrated by a communication network example.

\section{Control Applications for Complex Industrial Systems}

Thin coal seam mining is significant but challenging as it is subjected to small working space, low-level automation and drilling deviation. In the work authored by Ji and Liu [12], an integration of nonlinear adaptive backstepping controller and disturbance observer was addressed for position tracking control to achieve directional drilling on a coal auger. A stability analysis of the deviation control system was proved using Lyapunov stability theory. An electro-hydraulic servo displacement control experiment was set up to validate the proposed control strategy. The addressed control strategy would benefit technical breakthrough on horizontal directional drilling for thin coal seam mining.

Boost control for a variable geometry turbocharger-equipped diesel engine is a challenging task due to its strong coupling with the exhaust gas recirculation system and large delays. In the paper authored by Hu et al. [13], as one of the powerful model-free deep reinforcement learning algorithms, the deep deterministic policy gradient algorithm was used to track the target boost pressure under transient driving cycles. It showed that the proposed algorithm can achieve a satisfactory transient control performance from scratch by autonomously learning the interaction with environments. It is worthy to point out the proposed strategy can adapt to varying environment and hardware aging over time owing to its capability of self-learning on-line.

There is a growing demand for temperature control of thermal processing systems. In the paper authored by $\mathrm{Xu}$ et al. [14], a novel slow-mode-based control approach was presented for multipoint temperature control systems, where the temperature differences and the transient responses can be regulated, ensuring the outputs of the fast modes to follow that of the slow mode. The experiments were implemented under a DSP control platform. The proposed control algorithms were demonstrated to be effective compared with the conventional PI control methods.

In the paper authored by $\mathrm{Xu}$ et al. [15], a pole-zero cancellation method was proposed for temperature control in heating process systems, where the temperature differences and transient properties of all points can be adjusted by implementing dead time difference 
compensation and pole-zero cancellation with the feedforward reference model. Compared with conventional control approaches, the control efficiency of the proposed approach was well-demonstrated.

Voltage source converters can regulate active and reactive power rapidly, which has many applications such as in renewable energy systems and so on. In the paper by Jiang et al. [16], a novel optimal nonlinear adaptive control scheme was proposed to control voltage source converters. An extended state observer was used to estimate uncertain perturbations, and the perturbation compensation was implemented through state feedback. The proposed control strategy can ensure a consistent control performance even when operation conditions are varied. A hardware experiment was implemented to demonstrate the effectiveness of the proposed control design.

Steam/water loop is an important part of a steam power plant, which operates in harsh environments. As a result, it is challenging to design an effective controller to deliver satisfactory control performance for steam/water loop systems. Motivated by the above, Zhao et al., in [17] studied the feasibility of a distributed model predictive control strategy for steam/water loop systems. A multiple objective model predictive control approach was addressed to improve computing speed. The stability and convergence of the system under distributed model predictive control was discussed. Simulation tests were carried out on a steam/water loop system with five different sub-loops, demonstrating the effectiveness of the proposed control strategy.

The raceway reactor is the most common reactor on an industrial scale as it has advantages such as simplicity of operation and low cost for maintenance. In the work by Rodríguez-Miranda et al. [18], an event-based control architecture for PID controllers was presented, which aims to tune classical time-driven PI parameters for $\mathrm{pH}$ control, and then to build on event-based abilities while keeping the initial PI control design. The proposed event-based PI controller can achieve better performance by reducing the actuator effort and saving costs relevant to gas consumption, compared with the traditional on-off controllers.

In the paper authored by Ohrem et al. [19], control structure analysis and controller design were addressed for a novel multipipe separator, in order to enhance efficient production of hydrocarbons on the seabed in waters. PI controllers and model reference adaptive controllers were designed for different control loops. The proposed control methods were implemented and tested on a prototype of the separator concept, showing the effectiveness and drawing resultant conclusions.

In the paper authored by Zhang and Gao [20], an online data-driven approach was presented to improve the conversion efficiency of a refrigeration system under varying load conditions. A reinforcement learning approach was used to find out optimal actions using online data in the process level, and a coarse model was developed to evaluate action values. The actions were achieved as preset variables by implementing a single loop control. The effectiveness of the proposed approach was demonstrated by simulation studies on a test bed.

\section{Optimization for Complex Industrial Processes}

The paper authored by Tang et al., addressed a case study on optimization of the support design for a tunnel boring machine-an excavated coal mine roadway in Zhangii, China in [21]. An improved rock constitutive model of the roadway surrounding rocks was derived, and an updated failure criterion was presented based on laboratory rock tests. The proposed model and the failure criterion were used in simulation studies, and an optimal roadway support design was proposed based on simulation analyses. The feasibility and effectiveness of the support design was verified by in situ monitoring results.

Distributed generation systems play an important role in modern power networks. It is noticed that if the placement and sizing of the distributed generation systems were not selected properly, it would cause power system safety hazards. As a result, it is of significance to have a proper design for the placement and sizing of the distributed generators. In 
the work by Liu et al., in [22], an improved genetic algorithm was proposed to optimize the siting and sizing of the distributed generation units. The proposed optimization algorithm was demonstrated to be effective via various simulation experiments.

Han et al., proposed a novel optimization algorithm, by combining a simulated annealing algorithm-based Hopfield neural network algorithm and local scheduling rules, to solve the flexible flow shop scheduling problem with a public buffer [23]. The addressed local scheduling rules were used to control the moving process of the workpieces, reduce the production blockage, and improve the efficiency of the workpiece transfer. Based on a simulation using actual production data, the proposed method was proved to outperform the conventional methods in searching efficiency and optimization target.

In the work by Han et al. [24], a scheduling problem in a flexible flow shop with setup times was investigated, where practical constraints of the multiqueue limited buffer were taken into account in the addressed model. An improved compact genetic algorithm with local dispatching rules was presented, which was verified by using the real data from a bus manufacture production line, showing satisfactory performance.

In the work by Li et al., Aspen Plus software was used to simulate and optimize the separation of the aqueous acetonitrile solution by pressure swing distillation [25]. The total annual cost was used as the objective function, and the tray number, reflux ratio, and feeding position were the design variables to be optimized. Moreover, pressure swing distillation optimizations were compared with and without full-heat integration process. It concluded that it was more economical to separate the acetonitrile and water mixture by pressure swing distillation with full-heat integration.

In the paper authored by Cao et al. [26], reactive power optimization was investigated for large-scale power systems. A novel transfer bees optimizer was used, where Q-learning was employed to construct the learning mode of bees in order to improve the intelligence of bees through task division and cooperation. The simulation results demonstrated the proposed optimization algorithm possessed better convergent performance compared with the traditional artificial intelligence algorithms.

In the paper authored by Gao et al. [27], grouping semiconductor wire bonding equipment was investigated using processing task matching. Ahead of establishing the associated relationship between devices, a clustering by fast search and find of density peaks (CFSFDP) algorithm was addressed to cluster device attribute information and achieve the maximum number of groups of device sets, so that the resulting device groups can be obtained. The experimental results demonstrated that the improved equipment grouping method with CFSFDP algorithm outperformed the conventional methods. The equipment utilization of the bonding process segment was improved, and a good dynamic grouping was achieved so that the efficiency of the entire semiconductor production line was improved correspondingly.

Author Contributions: Conceptualization, Z.G.; writing—original draft preparation, Z.G.; writingrevision, Z.G., M.Z.Q.C., D.Z.; project administration, Z.G., M.Z.Q.C., D.Z. All authors have read and agreed to the published version of the manuscript.

Funding: There are no funding supports.

Acknowledgments: The guest editors would like to express great appreciation to all authors who contributed this Special Issue. Moreover, the guest editor team is deeply indebted to all reviewers for their constructive comments that helped shape this Special Issue.

Conflicts of Interest: The authors declare no conflict of interest.

\section{References}

1. Gao, Z.; Kong, D.; Gao, C. Modelling and control of complex dynamic systems: Applied mathematical aspects. J. Appl. Math. 2012. [CrossRef]

2. Gao, Z.; Nguang, S.; Kong, D. Advances in modelling, monitoring, and control for complex industrial systems. Complexity 2019. [CrossRef] 
3. Gao, Z.; Liu, X. An overview on fault diagnosis, prognosis and resilient control for wind turbine systems. Processes $2021,9,300$. [CrossRef]

4. Fu, Y.; Gao, Z.; Liu, Y.; Zhang, A.; Yin, X. Actuator and sensor fault classification for wind turbine systems based on fast Fourier transform and uncorrelated multi-linear principal component analysis techniques. Processes 2020, 8, 1066. [CrossRef]

5. Aslam, M.; Bantan, R.; Khan, N. Monitoring the process based on belief statistic for neutrosophic gamma distributed product. Processes 2019, 7, 209. [CrossRef]

6. Xie, B.; Yan, Z.; Du, Y.; Zhao, Z.; Zhang, X. Determination of Holmquist-Johnson-Cook constitutive parameters of coal: Laboratory study and numerical simulation. Processes 2019, 7, 386. [CrossRef]

7. Tang, Z.; Tang, L.; Zhang, G.; Xie, Y.; Liu, J. Intelligent setting method of reagent dosage based on time series froth image in zinc flotation process. Processes 2020, 8, 536. [CrossRef]

8. Cai, G.; Liu, X.; Dai, C.; Luo, X. Load state identification method for ball mills based on improved EWT, multiscale fuzzy entropy and AEPSO_PNN classification. Processes 2019, 7, 725. [CrossRef]

9. Sun, X.; Liu, Y.; Wang, J.; Li, J.; Sun, S.; Cui, X. Study on three-dimensional stress field of gob-side entry retaining by roof cutting without pillar under near-group coal seam mining. Processes 2019, 7, 552. [CrossRef]

10. Hu, P.; Lee, L. Community-based link-addition strategies for mitigating cascading failures in modern power systems. Processes 2020, 8, 126. [CrossRef]

11. Ibrahim, M.; Alsheikh, A. Automatic hybrid attack graph (AHAG) generation for complex engineering systems. Processes 2019, 7, 787. [CrossRef]

12. Ji, H.; Liu, S. Position deviation control of drilling machine using a nonlinear adaptive backstepping controller based on a disturbance observer. Processes 2021, 9, 237. [CrossRef]

13. Hu, B.; Yang, J.; Li, J.; Li, S.; Bai, H. Intelligent control strategy for transient response of a variable geometry turbocharger system based on deep reinforcement learning. Processes 2019, 7, 601. [CrossRef]

14. Xu, S.; Hashimoto, S.; Jiang, W.; Jiang, Y.; Izaki, K.; Kihara, T.; Ikeda, R. Slow mode-based control method for multi-point temperature control system. Processes 2019, 7, 533. [CrossRef]

15. Xu, S.; Hashimoto, S.; Jiang, W. Pole-zero cancellation method for multi input multi output (mimo) temperature control in heating process system. Processes 2019, 7, 497. [CrossRef]

16. Jiang, Y.; Jin, X.; Wang, H.; Fu, Y.; Ge, W.; Yang, B.; Yu, T. Optimal nonlinear adaptive control for voltage source converters via memetic salp swarm algorithm: Design and hardware implementation. Processes 2019, 7, 490. [CrossRef]

17. Zhao, S.; Maxim, A.; Liu, S.; Keyser, R.; Ionescu, C. Distributed model predictive control of steam/water loop in large scale ships. Processes 2019, 7, 442. [CrossRef]

18. Rodríguez-Miranda, E.; Beschi, M.; Guzmán, J.; Berenguel, M.; Visioli, A. Daytime/nighttime event-based PI control for the pH of a microalgae raceway reactor. Processes 2019, 7, 247. [CrossRef]

19. Ohrem, S.; Skjefstad, H.; Stanko, M.; Holden, C. Controller design and control structure analysis for a novel oil-water multi-pipe separator. Processes 2019, 7, 190. [CrossRef]

20. Zhang, D.; Gao, Z. Improvement of refrigeration efficiency by combining reinforcement learning with a coarse model. Processes 2019, 7, 967. [CrossRef]

21. Tang, B.; Cheng, H.; Tang, Y.; Zheng, T.; Yao, Z.; Wang, C.; Rong, C. Supporting design optimization of tunnel boring machinesexcavated coal mine roadways: A case study in Zhangji, China. Processes 2020, 8, 46. [CrossRef]

22. Liu, W.; Luo, F.; Liu, Y.; Ding, W. Optimal siting and sizing of distributed generation based on improved nondominated sorting genetic algorithm II. Processes 2019, 7, 955. [CrossRef]

23. Han, Z.; Han, C.; Lin, S.; Dong, X.; Shi, H. Flexible flow shop scheduling method with public buffer. Processes $2019,7,681$. [CrossRef]

24. Han, Z.; Zhang, Q.; Shi, H.; Zhang, J. An Improved compact genetic algorithm for scheduling problems in a flexible flow shop with a multi-queue buffer. Processes 2019, 7, 302. [CrossRef]

25. Li, J.; Wang, K.; Lian, M.; Li, Z.; Du, T. Process simulation of the separation of aqueous acetonitrile solution by pressure swing distillation. Processes 2019, 7, 409. [CrossRef]

26. Cao, H.; Yu, T.; Zhang, X.; Yang, B.; Wu, Y. Reactive power optimization of large-scale power systems: A transfer bees optimizer application. Processes 2019, 7, 321. [CrossRef]

27. Gao, Z.-J.; Si, W.; Han, Z.; Peng, J.; Qiao, F. Grouping method of semiconductor bonding equipment based on clustering by fast search and find of density peaks for dynamic matching according to processing tasks. Processes 2019, 7, 566. [CrossRef] 